\title{
Recombinant MIP1-alpha Variant ECI301
}

National Cancer Institute

\section{Source}

National Cancer Institute. Recombinant MIP1-alpha Variant ECI301. NCI Thesaurus. Code C98294.

A recombinant form of a human macrophage inflammatory protein-1 alpha (MIP1-alpha) with a substitution of aspartate to alanine at position 26 , with potential immunomodulating and radiotherapy potentiating activity. Intravenous administration of recombinant MIP1-alpha variant ECI301 after local tumor irradiation enhances the antitumor effect of ionizing radiation at the irradiated site as well as the antitumor effect at non-irradiated tumor sites (known as the abscopal effect). The abscopal effect appears to be attributed to this agent's ability to recruit and activate leukocytes, such as monocytes, dendritic cells, natural killer cells and T lymphocytes, thereby initiating an antitumor immune response against cancer cells. MIP1-alpha, also known as chemokine (C-C motif) lig and 3, is a ligand for the chemokine receptors CCR1, CCR4 and CCR5 that are involved in immune and inflammatory responses. 